\title{
Desafíos de una nueva justicia dialógica ante la vigencia del ius constitutionale commune en América Latina
}

\section{Challenges of a new dialogic justice before the validity of ius constitutionale commune in Latin America}

https://doi.org/10.15332/iust.v0i17.2420

\section{Christian Benítez Núñez}

Abogado, maestro en Derecho, PhD. en Derecho y Globalización por la Facultad de Derecho y Ciencias Sociales de la Universidad Autónoma del Estado de Morelos, México; estudios de posdoctorado en la Facultad de Derecho, Ciencias Politicas y Sociales de la Universidad Nacional de Colombia, Bogotá; candidato a investigador nacional SNI-CONACYT; estancia post doctoral Universidad Nacional de Colombia-sede Bogotá Correoelectrónico: chbenitezn@unal.edu.co

\begin{abstract}
Resumen
La presente investigación parte de un método histórico-comparativo sobre el estado de derecho y su evolución hasta el siglo XXI, así como de sus implicaciones formales y sustanciales en un contexto de globalización tendiente a la materialización de los derechos humanos, a partir de su exigibilidad en sede jurisdiccional tanto nacional como internacional. Concluyendo que el diálogo entre cortes constitucionales, la incorporación de la figura del estado de cosas inconstitucionales y la adopción de la metodología establecida en el Protocolo de San Salvador, establecen las bases para una adecuada respuesta a los problemas estructurales que tienen lugar en América Latina.
\end{abstract}

Palabras clave: Justicia dialógica, estado de cosas inconstitucionales, ius constitutionale commune

\section{Abstract}

This research is based on a historical-comparative method on the rule of law and its evolution up to the 21 st century, as well as its formal and substantial implications in a context of globalization tending to the materialization of human rights from its enforceability in jurisdictional seat, both national and international. Concluding that the dialogue between constitutional courts, the incorporation of the figure of the unconstitutional state of affairs and the adoption of the methodology established in the Protocol of San Salvador, establish the bases for an adequate response to the structural problems that take place in Latin America.

Keywords: Dialogical justice, unconstitutional state of affairs, ius constitutionale commune 


\section{Résumé}

Cette recherche est basée sur une méthode historico-comparative sur l'état de droit et son évolution jusqu'au XXIe siècle, ainsi que sur ses implications formelles et substantielles dans un contexte de mondialisation tendant à la réalisation des droits de l'homme sur la base de son applicabilité dans siège juridictionnel national et international. Concluant que le dialogue entre les cours constitutionnelles, l'incorporation de la figure de la situation inconstitutionnelle et l'adoption de la méthodologie établie dans le Protocole de San Salvador, jettent les bases d'une réponse adéquate aux problèmes structurels qui se posent en Amérique latine.

Mots-clés: justice dialogique, situation inconstitutionnelle, commune constitutionnelle 


\section{Desafíos de una nueva justicia dialógica ante la vigencia del ius constitutionale commune en América Latina ${ }^{1}$}

Christian Benítez Núñez

\section{INTRODUCCIÓN}

Nos encontramos en tiempos de constantes transformaciones en diversas áreas políticas, jurídicas y económicas, que inevitablemente impactan en la sociedad y en sus relaciones con el poder, tanto en el ámbito nacional como en el internacional; ello me lleva a afirmar una impresión primera: lo único que permanece es el cambio y no en pocas ocasiones se presenta la dificultad de acompasarlo.

En este contexto evolutivo es que surge un concepto político-jurídico que se le identifica como democracia constitucional, el cual implica un modelo de Estado contemporáneo que ha sido adoptado en los países que se reconocen como democráticos, toda vez que cobra especial relevancia la dimensión sustancial -conformada por las libertades y los derechos sociales-, junto a la dimensión formal -derechos civiles y políticos-.

Ahora bien, las coincidencias en los órdenes jurídicos en cada país permiten identificar la adopción de este modelo de Estado, aunado a ello, el reconocimiento de un gran número de instrumentos internacionales ha conformado un ius commune latinomericano, lo que nos permite crear puentes de diálogo entre diversos operadores jurídicos para superar las graves violaciones a los derechos humanos.

Los desarrollos jurisprudenciales como es el estado de cosas inconstitucional, junto con la implementación de indicadores especificados, tanto en el ámbito universal como regional, permiten trazar la hoja de ruta para la reforma y control de marcos normativos que impidan la satisfacción de derechos, así como el redireccionamiento de las políticas públicas hacia la consecución del bien común, y la transformación en la cultura jurídica, tanto en la judicatura como en la propia academia, para abandonar teorías anacrónicas que no corresponden con los retos del presente.

1 Artículo resultado de investigación CONACYT durante el Programa de Estancias Posdoctorales en el Extranjero 2018-1, en trabajo colaborativo de los grupos de Investigación Escuela de Derecho Penal "Nullum Crimen Sine Lege" un, reconocido y clasificado en A Colciencias, 2015 Universidad Nacional de Colombia, COL0078909; el Centro Interamericano de Investigación en Derechos Humanos y Litigio Estratégico CIIDHLEX A.C.; y la Universidad Autónoma del Estado de Morelos. 


\section{EVOLUCIÓN DEL MODELO DE ESTADO LEGISLATIVO DE DERECHO AL MODELO DE DEMOCRACIA CONSTITUCIONAL}

He de comenzar el desarrollo de la presente investigación, estableciendo como punto de partida un breve contexto en torno a lo que debemos entender por Estado, los elementos que comprende, así como los fines que pretende alcanzar. No obstante, debo aclarar que ello no implica un estudio pormenorizado desde la antigüedad hasta nuestros tiempos, sino más bien, el abordaje de los puntos esenciales.

Ahora bien, un concepto que resulta mayoritariamente aceptado es aquel que describe al Estado como "una multitud de hombres, que están situados en una parte rigurosamente delimitada de la superficie terrestre, bajo un poder organizado, esto es, ordenado, y por cierto, ordenado jurídicamente" (Kelsen, 1992, p. 133). Dicho concepto permite identificar los elementos constitutivos: población, territorio y poder -y la condición de sujetarse a un orden jurídico-. Además, desde una perspectiva formal Norberto Bobbio (1989) señala que en un territorio determinado debe existir un poder capaz de tomar decisiones y emitir mandatos obligatorios, y por lo tanto, que sean obedecidos - en su mayoría- por los destinatarios.

Conviene subrayar que en un Estado -al menos democrático-, el ejercicio del poder debe encontrarse orientado a la materialización de ciertos fines; no obstante, algunas veces se pierde el rumbo para el cual fue constituido. Por ejemplo, Francis Fukuyama (2004) afirma que los Estados poseen una variedad de funciones que pueden orientarse tanto hacia una dimensión positiva como negativa, toda vez que el mismo poder coactivo que les permite defender derechos de sus ciudadanos, también en algunos casos les permite vulnerarlos.

Por consiguiente, no se debe perder de vista que la legítima función del Estado debe perseguir el bien común y la armonía social:

La función del Estado consiste, pues, en la organización y activación autónomas de la cooperación social-territorial, fundada en la necesidad histórica de un status vivendi común que armonice todas las oposiciones de intereses dentro de una zona geográfica, la cual, en tanto exista un Estado mundial, aparece delimitada por otros grupos territoriales de dominación de naturaleza semejante (Heller, 2015).

En pocas palabras, el Estado representa una organización social que se encuentra en un territorio plenamente delimitado, en el que surge por consenso un poder facultado para ordenar y hacer cumplir sus órdenes con apego a un orden jurídico (Huertas, Torres, \& Díaz, 2011), el cual contiene límites formales y sustanciales orientados a la satisfacción de las necesidades individuales y colectivas. Afirmo que el Estado ha evolucionado permanentemente a lo largo de los siglos y es en este en donde el elemento constitutivo denominado poder cobra especial relevancia, ya que resulta imprescindible para alcanzar el bien común dentro de una sociedad; de ahí que las propias reglas impuestas por los diversos sistemas jurídicos de naturaleza democrática han pretendido limitar y encausar su acción. 
Por ejemplo, si nos trasladamos a los documentos fundamentales donde se establece la necesidad de limitar al poder, resulta imprescindible hacer mención de la Declaración de los Derechos del Hombre y del Ciudadano que data de 1789, en cuyo artículo 16 se estableció que "[t]oda sociedad en la que la garantía de los derechos no esté asegurada, ni determinada la separación de poderes, no tiene constitución" (Jellinek, 2003). Este resulta ser el fundamento del Estado moderno en el que el poder se desconcentra a partir de una división específica para que pueda ser controlado con base en sus fines.

Cabe resaltar que la condición de la necesaria garantía de los derechos representa un avance significativo en la reivindicación de estos. Tal afirmación cobra mayor fuerza con la precisión de Georg Jellinek al respecto, de que, en este documento, es por vez primera, que se forma en el derecho positivo la noción de los derechos subjetivos del individuo del Estado frente a este, destacando lo siguiente:

[B]ajo su influjo se ha formado la noción de los derechos subjetivos públicos del individuo en el derecho positivo de los Estados del Continente Europeo. La literatura jurídico-política sólo conocía hasta entonces derechos de los Jefes de Estado, privilegios de clase, de los particulares o de ciertas corporaciones, no manifestándose los derechos generales de los súbditos sino bajo la forma de deberes del Estado, sin constituir para los individuos títulos jurídicos caracterizados (Jellinek, 2003).

Volviendo al tema que nos ocupa, he de advertir que las condiciones establecidas en la Declaración de los Derechos del Hombre y del Ciudadano -además de representar la base de lo que ahora se denomina constitucionalismo- fueron también advertidas por Montesquieu (2015), al sostener que la separación de poderes encuentra su justificación atendiendo a que la concentración de las funciones correspondientes al poder legislativo y al poder ejecutivo en una misma persona, pondrían en riesgo tanto la libertad como la confianza, ya que aquel en quien se concentra el poder podría expedir leyes tiránicas y ejecutarlas tiránicamente. Por otra parte, tampoco existirían condiciones para el desarrollo de la libertad si el poder judicial no estuviese deslindado de los poderes legislativo y ejecutivo, ya que podría disponer arbitrariamente de esta y además de la propia vida, impidiendo con ello el desarrollo del individuo y de la sociedad en la que se inserta.

Cabe señalar que el contenido de dicha cláusula ha sido recepcionado por las constituciones contemporáneas, dando lugar al reconocimiento de un amplio catálogo de derechos humanos en lo que se conoce como parte dogmática, así como la división de poderes y su estructuración más o menos delimitada en aquella que se denomina parte orgánica. De ahí la importancia de una constitución como cimiento de todo orden normativo positivo y de uno de sus principales atributos, la rigidez, que permite la prolongación de su vigencia a lo largo del tiempo. Así, por ejemplo, Ferdinand Lasalle (2009) hizo hincapié en que la constitución y la ley, si bien es cierto, tienen una esencia genérica común; la primera debe ser algo mucho más sagrado, más firme y más inconmovible que la ley, ya que es el fundamento de las otras leyes. 
Deseo subrayar que un Estado que adopta una constitución bajo las condiciones previamente señaladas, puede denominársele Estado constitucional. Además, esta adopción de modelo de Estado representa una organización política, liberal y garantista, que reconoce a la constitución como un límite al poder político (Guastini, 2001). Por tal motivo, debe tenerse en cuenta que la subordinación de los poderes a los derechos reconocidos constitucionalmente implica tanto una obligación de respeto como de garantía, traduciéndose en el ineludible margen de actuación las autoridades estatales.

Al respecto conviene decir que la evolución de modelo de Estado constitucional tiene sus antecedentes al menos en dos modelos previos -sobre los cuales únicamente resaltaré los aspectos más fundamentales-, y que parten de la validez del propio derecho, recordando que este condiciona dentro de un Estado, no solo al poder, sino además las propias situaciones que tienen lugar en la vida social individual y colectiva.

El primer modelo es descrito por Luigi Ferrajoli (2014) como el derecho jurisprudencial premoderno, y que al no existir un monopolio de la producción del derecho, representó "un patrimonio de máximas, categorías, principios y precedentes judiciales transmitido por la cultura y la práctica jurisprudencial y doctrinal” (p. 18), cuya norma de reconocimiento dependió de la justicia y verdad intrínsecas, y que puede resumirse en la frase Veritas, non auctoritas facit legem.

El segundo modelo corresponde al legislativo o páleo-positivista, caracterizado por el monopolio estatal de la producción normativa, teniendo como base el principio de legalidad, y cuya validez de las normas se justificó a partir de su positividad y no de su intrínseca racionalidad, modelo reducido a la frase Auctoritas, non veritas facit legem (Ferrajoli, 2014, pp. 18-19).

Habría que decir también, que la historia nos ha demostrado que bajo este modelo legislativo se han cometido las más grandes atrocidades (Huertas, Amaya, \& Malte, 2013), lamentablemente con estricto apego a la ley -sin que la justicia o la racionalidad tengan lugar-, estableciendo estados de excepción con cierta regularidad para justificar tanto las decisiones como actos más antiliberales dentro de un aparente modelo democrático. Una acertada crítica a dicho modelo la encontramos en la obra de Gustavo Zagrebelsky (2005) al señalar que en el Estado legislativo de derecho se reduce todo lo que pertenece al mundo del derecho - como lo son los derechos y la justicia-, a lo dispuesto por la ley, encontrando aún en nuestros tiempos actuales, residuos que solamente se pueden explicar a través de la fuerza de la tradición.

Por lo que se refiere al tercer modelo, encontramos al Estado constitucional de derecho o neo-positivista, en el que se toma en cuenta para la validez del derecho, no solo las formas de producción, sino, además, su contenido:

[M]ientras la existencia o vigencia de las normas continúa dependiendo de su forma de producción, cuya norma de reconocimiento sigue siendo el viejo principio de legalidad formal, su validez depende también de su sustancia o contenido, cuya norma de reconocimiento consiste en el principio de legalidad sustancial que la vincula a la coherencia con los principios y los derechos constitucionalmente establecidos (Ferrajoli, 2014, p. 19). 
Es en este contexto que Gustavo Zagrebelsky (2005) da cuenta de que la ley por vez primera se encuentra sometida a unas condiciones de adecuación y, por lo tanto, de subordinación a los contenidos constitucionales. En otras palabras, la ley ha quedado destronada, mientras que el principio de constitucionalidad debe asegurar el objetivo de unidad y paz entre las sociedades divididas en su interior.

Considero necesario subrayar que todo lo dicho en líneas anteriores, guarda una estrecha relación con la necesidad de controlar al poder y con el respeto y garantía de los derechos humanos. Por lo tanto, encuentro razones suficientes para abandonar la creencia a priori de que el poder y los derechos guardan una esencia antagonista, más bien considero que representan una simbiosis dentro del propio Estado, y que incluso, el primer elemento resulta indispensable para la materialización de tales derechos.

Aquí podría afirmar que el elemento que sirve de puente entre el poder y los derechos, lo representa la democracia, la cual es definida por Hermann Heller (2015, p. 224) como una estructura de poder construida de abajo hacia arriba, en la que rige el principio de soberanía del pueblo; concibiéndose a este como un principio polémico de la división política del poder, opuesto al principio de la soberanía del dominador.

De manera similar, Norberto Bobbio (2005) precisa que el término democracia se presenta de manera sintetizada como "el poder público activo, informado, consciente de sus derechos." Asimismo, sostiene que este término se emplea para designar una de las formas en las que se ejerce el poder político, destacando que esta forma se caracteriza por ser el pueblo el titular del poder (Bobbio, 1989, p. 188). Indiscutiblemente, el término democracia implica el poder del pueblo o de la sociedad, y esto tiene sentido, toda vez que en palabras del propio Norberto Bobbio (2003) resultaría "improbable que un Estado no liberal pueda asegurar un correcto funcionamiento de la democracia y, por otra parte, es poco probable que un Estado no democrático sea capaz de garantizar las libertades fundamentales".

Otro aspecto, que no se debe dejar pasar inadvertido, corresponde al desarrollo de las ideas jurídicas y políticas de Luigi Ferrajoli (2012), quien identifica la existencia de dos dimensiones de la democracia; dimensión formal o procedimental, desarrollada a partir de las formas el quién y los procedimientos idóneos el cómo, para garantizar la voluntad popular, independientemente de sus contenidos; y una dimensión sustancial, establecida por los derechos humanos, el principio de igualdad, entre otros.

Ahora bien, ambas dimensiones tanto formal como sustancial, no se contraponen, sino que se complementan, dando como resultado el modelo denominado democracia constitucional, que de acuerdo con Luigi Ferrajoli (2011) representa un modelo normativo multidimensional que corresponde a diversas clases de derechos fundamentales:

- Democracia política: asegurada por las garantías de los derechos políticos.

- Democracia civil: asegurada por las garantías de los derechos civiles.

- Democracia liberal: asegurada por las garantías de los derechos de libertad.

- Democracia social: asegurada por las garantías de los derechos sociales. 
Recientemente, Luigi Ferrajoli (2014) ha llegado a la conclusión de que los derechos políticos y civiles a los cuales denomina formales o instrumentales, sirven para fundamentar la legitimidad de la forma de las decisiones en la esfera política y en la esfera económica; mientras que los derechos de libertad y sociales a los que denomina sustanciales o finales, sirven para fundar la legitimidad de la sustancia de las decisiones. Tales dimensiones representan la base del modelo de democracia constitucional en virtud del cual, ningún poder puede derogar o desconocer los derechos fundamentales y demás principios establecidos por la propia Constitución.

Lo que aquí me interesa resaltar es cómo el desarrollo conceptual del Estado ha ido ajustándose de acuerdo con su complejo contexto contemporáneo; sin que tal circunstancia haya provocado el abandono de la esencia liberal de finales de siglo XVIII; a mi parecer, resulta un perfeccionamiento, una puntualización a nivel teórico de las dimensiones que el Estado mismo implica a día de hoy. La democracia constitucional representa entonces un modelo integral del Estado, ya que al considerar sus dimensiones formales y sustanciales desarrolla obligaciones ineludibles por parte de las autoridades, tendientes a alcanzar el verdadero orden social por el cual fue creado el Estado y dotado de poder, para servir a la comunidad y no para servirse de esta.

En esa línea evolutiva, la democracia constitucional ha sido adoptada por un gran número de países en el mundo, a través del reconocimiento de un amplio catálogo de derechos humanos tanto de fuente nacional como supranacional. Ello permite identificar unas coincidencias que en el plano teórico actual denominamos ius commune, es decir, un derecho común; permitiendo el estudio de la efectividad de tales derechos a través de los marcos normativos y desarrollos jurisprudenciales, tal y como demostraré en los siguientes apartados.

\section{La vigencia de un ius commune latinoamericano}

A partir de la recepción de la Convención Americana de Derechos Humanos (en adelante $\mathrm{CADH}$ ) en cada orden interno, mediante las disposiciones constitucionales de cada Estado-parte, se construye un nuevo orden jurídico regional desde dos perspectivas; la primera internacional, ya que es en esta sede donde nace la CADH y, posteriormente, una sede nacional, que se sujeta a su contenido.

Una vez planteado lo anterior, son claros los fines que persigue esta comunidad en vías de desarrollo, partiendo del respeto a los derechos humanos y el reconocimiento de la dignidad de las personas, el ius commune latinoamericano se propone tres objetivos:

1. Avanzar en el respeto de tres principios: derechos humanos, Estado de derecho, democracia, partiendo de la premisa de la insuficiencia del espacio estatal, sin que esto implique el desplazamiento del Estado como institución primordial del derecho público.

2. Concretizar el Estado abierto a partir de las cláusulas de apertura: al derecho internacional, a las instituciones internacionales y la práctica del derecho 
comparado, basándose en la incapacidad del Estado aislado para superar los problemas del siglo XXI. Traduciéndose esa apertura en progreso.

3. La consolidación de instituciones internacionales fuertes, eficientes y legítimas: que coadyuven con la protección de derechos humanos desde una perspectiva con mayor alcance que la que podría tener un Estado-nación bajo el principio de universalismo (Von Bogdandy, 2013).

Fines que en mayor o menor medida se han ido alcanzando en los últimos años, por lo que resulta innegable que aquellos países de la región que fueron sometidos por las dictaduras en el siglo XX, como Chile con Augusto Pinochet, Panamá con Manuel Antonio Noriega, Nicaragua con Anastasio Somoza o Paraguay con Alfredo Stroessner, han transitado en las últimas décadas hacia un Estado democrático, en el que se ha robustecido el catálogo de derechos humanos mediante la recepción de instrumentos internacionales.

La vigencia de tal parámetro de regularidad implica un cambio de paradigma, toda vez que el constitucionalismo contemporáneo ha redefinido a la democracia en su dimensión formal y sustancial; la rigidez constitucional entendida como norma supraordenada a la legislación ordinaria ha traído como consecuencia, a nivel interno, la sujeción de los poderes públicos a la ley constitucional; y a nivel externo, los Estados se encuentran sometidos al pactum subiectionis del derecho internacional (Ferrajoli, 2013).

Ahora bien, solo para evidenciar el reconocimiento amplio de derechos como rasgo esencial de este nuevo constitucionalismo, he de señalar cuatro países de la región latinoamericana en los que se encuentra vigente un bloque de constitucionalidad, tal y como se expone a continuación:

Tabla 1. Vigencia del Bloque de constitucionalidad en los países latinoamericanos

\begin{tabular}{|c|c|c|c|}
\hline PAÍS & AÑO & ARTÍCULO(S) & CONTENIDO \\
\hline Argentina & 1994 (Reforma) & 75 inciso 22 & $\begin{array}{l}\text { Reconoce la vigencia de un bloque de constitucio- } \\
\text { nalidad conformado por un amplio número de trata- } \\
\text { dos, entre ellos la CADH. }\end{array}$ \\
\hline Colombia & 1991 & 93 & $\begin{array}{l}\text { Los tratados y convenios internacionales que re- } \\
\text { conocen los derechos humanos y que prohíben su } \\
\text { limitación en los estados de excepción, prevalecen } \\
\text { en el orden interno. }\end{array}$ \\
\hline México & 2011 (Reforma) & $1^{\circ} \mathrm{y} 133$ & $\begin{array}{l}\text { Todas las personas gozarán de los derechos recono- } \\
\text { cidos en esta Constitución y en los tratados interna- } \\
\text { cionales de los que el Estado mexicano sea parte. La } \\
\text { Constitución, las leyes del Congreso de la Unión y } \\
\text { los tratados serán la ley suprema de toda la Unión. }\end{array}$ \\
\hline Perú & 1993 & $3^{\circ}$ y 55 & $\begin{array}{l}\text { Los tratados celebrados por el Estado y en vigor for- } \\
\text { man parte del derecho nacional. }\end{array}$ \\
\hline
\end{tabular}

Fuente: Elaboración propia a partir del estudio de cada una de las constituciones aquí señaladas. 
Resulta entonces que los derechos humanos que comprenden el bloque de constitucionalidad o parámetro de regularidad, representan lo que Ernesto Garzón Valdés (2003) denominó coto vedado y que consiste en "aquellos intereses y deseos primarios de las personas que no pueden ser afectados". De manera similar, Luigi Ferrajoli (2013, pp. 102-103) lo identifica como la esfera de lo indecidible, es decir, el conjunto de principios que se encuentran sustraídos a la voluntad de las mayorías, y que tampoco puede dejar de ser decidido, vinculando no solo a los poderes públicos, sino además, a los poderes privados.

Es de advertirse que, de tales disposiciones constitucionales y convencionales, se desprende una serie de obligaciones que requieren participación de cada uno de los poderes estatales. Tal afirmación tiene como fundamento, en el caso mexicano el párrafo tercero del artículo $1^{\circ}$, que establece que todas las autoridades en el ámbito de sus competencias tienen la obligación de respetar y garantizar derechos humanos. Ahora bien ¿de qué manera se puede cumplir eficazmente con tales obligaciones? Atendiendo a la experiencia comparada, no me cabe la menor duda que a través de un activismo judicial que no solo ordene acciones al resto de los órganos estatales, sino además que coordine y supervise estas, con el objetivo de garantizar ese amplio catálogo de derechos vigente (Huertas, 2019).

Advierto, entonces, que esta construcción del ius commune requiere de un diálogo constante entra cada uno de los Estados que conforman la región y de estos con los organismos internacionales que se encargan de vigilar el respeto del marco jurídico supranacional. Resulta más que evidente que tanto Colombia como México han tenido la voluntad -al menos normativamente-de impulsar esa consolidación de un ius commune latinoamericano, y de igual manera un gran número de países de la región se encuentra en la misma disposición, en un primer momento, del respeto de los derechos humanos en el orden interno, como garantía primaria (Huertas \& Morales, 2013).

En ese sentido, el ius commune latinoamericano en derechos humanos, es un cuerpo normativo y de doctrina, que tiende a conformar una cultura jurídica común en el espacio latinoamericano y, como tal, se han de observar los siguientes retos:

1. Diálogo entre Cortes.

2. La oportuna intervención de la academia.

3. La implementación de una estrategia de difusión de esta nueva cultura jurídica latinoamericana.

Al respecto del primer reto, Diálogo entre Cortes, encontramos su justificación en que en el ámbito de protección de los derechos humanos existe una tendencia de establecer entre el orden nacional e internacional una relación de influencia recíproca y de mutuo enriquecimiento, capaz de producir éxitos de gran relevancia dogmática y práctica (Rolla, 2002) y, por lo tanto, tal como lo señala Serna (2012) la existencia de un pluralismo interpretativo de una misma fuente ha llevado a intentos por encontrar modelos y concepciones que induzcan a entrar en una dinámica más 
de cooperación y diálogo, más que de competencia y conflicto. Convirtiéndose este diálogo en un referente jurisdiccional para garantizar la efectividad de los derechos humanos, ius constitutionale commune en América Latina.

De igual manera, por cuanto a la oportuna intervención de la academia en la construcción de una ciencia del derecho público integral, es hoy tan necesaria, porque como lo precisa el Dr. Lucio Pegoraro (2011), la intensa circulación de modelos impide el estudio individual de las instituciones de derecho público sin tener en cuenta las influencias que provienen del exterior, así como la adquisición de visiones comunes en el ámbito legislativo y, sobre todo, el jurisprudencial, además de cada vez mayor uniformidad del tejido normativo y eficacia que se desprende de las convenciones internacionales en materia de derechos humanos. Por lo tanto, únicamente bajo una mirada holística y crítica del nuevo derecho público y el desarrollo de una ciencia propia, así como de la implementación de una estrategia de difusión, se podrá hacer frente a los retos en materia de protección de derechos humanos en sede regional.

He de concluir este apartado, precisando que la construcción de un ius commune latinoamericano en materia de derechos humanos representa un proyecto en constante evolución que implica una cooperación de los Estados-parte que han ratificado la $\mathrm{CADH}$, siendo una verdad irrefutable que el Estado-nación desde una perspectiva individualista no será capaz de enfrentar de manera eficaz y eficiente, los grandes retos que implica el reconocimiento de la dignidad humana y la protección de los derechos de todas las personas.

\section{La justicia dialógica y el estado de cosas inconstitucional}

¿Cómo trasladar de la letra a la realidad, todo el contenido del corpus iuris en materia de derechos humanos? Para responder a tal interrogante, he de señalar que el reconocimiento de los derechos humanos de fuente internacional ha traído consigo una transformación por cuanto a las formas en que los ciudadanos acuden formalmente ante las Cortes para la exigibilidad de tales derechos, implicando con ello una intervención judicial en la política, abandonando -lentamente- el formalismo jurídico y demostrando cierto activismo judicial (Huneeus, Couso, \& Rachel, 2010).

En ese contexto en el que el jurista argentino Roberto Gargarella ha realizado una serie de estudios sobre el fenómeno identificado como Justicia Dialógica que, entre otros puntos, implica una novedosa práctica de diálogo que surge en América Latina, derivado de los litigios de tipo estructural y que es impulsado por los tribunales superiores, práctica que versa sobre problemas públicos y graves violaciones de derecho, en las que en audiencias públicas participan las partes involucradas, tanto estatales como representantes de la sociedad civil. Y por otra parte, la denominada "forma de revisión judicial débil" junto con la "revisión política de constitucionalidad" que ha traído consigo un nuevo tipo de relación dialógica entre tribunales y el poder legislativo, sin embargo, requiriéndose una estructura institucional que esté al servicio de las razones para superar las tensiones entre poderes (Gargarella, 2014). 
Con lo anterior, podemos observar que existe un sistema de pesos y contrapesos vigente, pero distinto al concebido originalmente, toda vez que existen espacios de diálogo y de construcción de lo que he denominado "Tétrada Democrática" conformada por los poderes ejecutivo, legislativo, judicial y la sociedad. Sin embargo, he de advertir que esta tendencia de diálogo está dando sus primeros pasos en América Latina, toda vez que cada uno de los poderes establecidos presenta cierto rechazo a la "invasión" de sus competencias, aún y cuando tales intervenciones tiendan a fortalecer a la democracia constitucional.

Las tensiones entre poderes siempre existirán, así lo sostiene Dominique Rousseau (2013), cuando señala que las relaciones conflictivas más recurrentes son aquellas con el poder político, "el cual denuncia regularmente el inadmisible "gobierno de los jueces" cada vez que una jurisdicción constitucional sanciona su obrar". Ahora bien, independientemente del país, los conflictos entre la Corte Constitucional y los poderes ejecutivo y legislativo resultan ser inevitables, ya que con el control constitucional el régimen tradicional de fabricación de la ley se trastorna, toda vez que existe un nuevo régimen concurrencial, sin embargo, tales conflictos son calificados como positivos, ya que a partir de ellos se crea una nueva forma de democracia.

Siguiendo esta línea, González Cuervo (2013) ha precisado que la Corte Constitucional colombiana se ha topado con situaciones de vulneración generalizada y sistemática de los derechos fundamentales ${ }^{2}$, que requieren del restablecimiento de la vigencia de los derechos para cumplir con el mandato constitucional. En ese sentido, en no pocas ocasiones sus acciones se entremezclan con competencias del poder ejecutivo, ya que las deficiencias del funcionamiento de las instituciones, la ausencia de aplicación correcta de las políticas públicas y las dificultades administrativas han conducido a la Corte a pronunciarse a través de sus sentencias y jurisprudencia para corregir tales deficiencias en el respeto y garantía de los derechos humanos.

Ciertamente, se ha evolucionado de un efecto inter partes a un efecto que en Colombia se ha denominado inter comunis, atacando los problemas a través de órdenes complejas que van dirigidas a los problemas estructurales que subyacen en el quebrantamiento concreto del derecho y que teniendo en cuenta el impacto financiero que provoca este tipo de resoluciones, se creó la figura del Incidente de impacto fiscal a través del cual el Procurador General de Justicia establece un diálogo con la Corte Constitucional, con el objetivo de que pondere respecto del impacto que tendría la decisión en las finanzas públicas.

Aun y cuando parecería un fenómeno novedoso, en el constitucionalismo norteamericano ya se había adelantado al constitucionalismo latinoamericano desde hace varias décadas. Prueba de ello son los estudios realizados por Owen Fiss (2007) a partir del activismo de la Corte Warren y sus retrocesos a partir de 1976 con las cortes Burger y Rehnquist. Independientemente de su génesis, lo que resulta ser imprescindible es que la

2 Tal situación se puede observar en las sentencias T-260 de 2008, T-153 de 1998, T-025 de 2004 de la Corte Constitucional de Colombia. 
Constitución establece la estructura estatal, así como los valores que la habrán de limitar, valores que resultan ser ambiguos y por lo tanto requieren ser dotados de significado especifico, lo cual se pretende alcanzar a través del proceso judicial; es entonces que el juez constitucional asume la tarea de reestructurar la organización pública para combatir la amenaza de los valores constitucionales.

Una vez precisado todo lo anterior, la Justicia dialógica parte de la necesidad de contrarrestar problemas estructurales. En ese sentido, la identificación de tales problemas la llevaremos a cabo a partir de los indicadores que César Rodríguez Garavito (2014) expone:

1. Afectación de un gran número de personas que denuncian violación de un conjunto de derechos.

2. Que los organismos principalmente estatales, ya sea por acción o por omisión contribuyan a la violación de derechos.

3. La protección de los derechos de las personas afectadas, impliquen la actuación de una pluralidad de órganos del Estado.

De tal manera que, una sentencia estructural representa la respuesta a problemas estructurales, dando pauta al surgimiento de lo que en el derecho comparado se conoce como leading case; es decir, sentencias hito que a su vez, permiten desarrollar líneas jurisprudenciales, haciendo frente a las violaciones de derechos humanos. Cabe señalar que tales líneas jurisprudenciales son desarrolladas tanto en sede nacional -tribunales constitucionales- como en sede internacional -Corte Interamericana, Tribunal Europeo, etc.- Por cuanto corresponde a la jurisdicción nacional, tanto Argentina como Colombia han enfrentado problemas estructurales relacionados con el sistema carcelario, el acceso a los servicios de salud, el desplazamiento forzado interno, entre otros; mientras que la Corte Interamericana ha desarrollado líneas jurisprudenciales relacionadas con la tortura, la ejecución extrajudicial, desaparición forzada, entre otros.

De ahí que mi propuesta se desarrolle en el sentido de un concepto de "Justicia dialógica multidireccional", cuyo centro del proceso dialógico sea el Tribunal Constitucional Nacional, tal y como se representa a continuación:

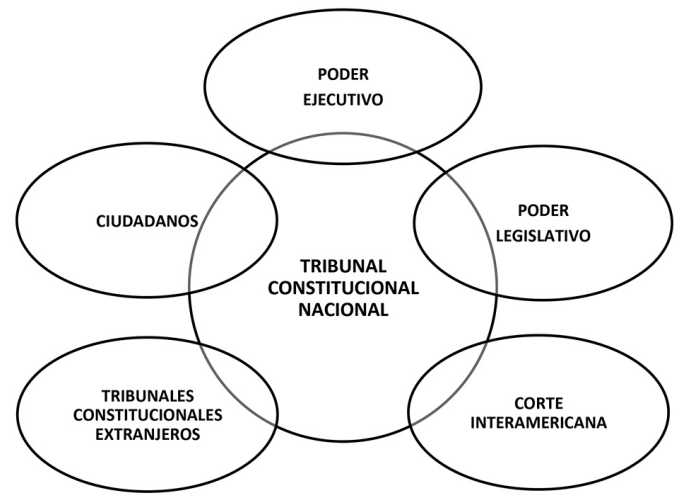

Fuente: Elaboración propia. 
Al llegar a este punto, he de resaltar la importancia de la figura jurisprudencial denominada estado de cosas inconstitucional, desarrollada por la Corte Constitucional de Colombia, la cual -desde mi perspectiva- representa una clave para la consolidación del ius commune latinoamericano a partir de su adopción por parte del resto de las altas Cortes. Dicha figura tiene por objetivo contrarrestar violaciones a los derechos humanos que requieren de un tratamiento diferenciado por parte de los órganos e instituciones del Estado en su conjunto, así la Corte Constitucional precisó lo siguiente:

[E]sta Corporación ha hecho uso de la figura del estado de cosas inconstitucional con el fin de buscar remedio a situaciones de vulneración de los derechos fundamentales que tengan un carácter general -en tanto que afectan a multitud de personas-, y cuyas causas sean de naturaleza estructural -es decir que, por lo regular, no se originan de manera exclusiva en la autoridad demandada y, por lo tanto, su solución exige la acción mancomunada de distintas entidades (Sentencia T-153-98).

De lo anterior, la Corte Constitucional (Sentencia T-025/04) ha enumerado seis factores para tener en cuenta para la determinación de la existencia de un estado de cosas inconstitucional:

1. La vulneración masiva y generalizada de varios derechos constitucionales que afecta a un número significativo de personas;

2. la prolongada omisión de las autoridades en el cumplimiento de sus obligaciones para garantizar los derechos;

3. la adopción de prácticas inconstitucionales, como la incorporación de la acción de tutela como parte del procedimiento para garantizar el derecho conculcado;

4. la no expedición de medidas legislativas, administrativas o presupuestales necesarias para evitar la vulneración de los derechos.

5. la existencia de un problema social cuya solución compromete la intervención de varias entidades, requiere la adopción de un conjunto complejo y coordinado de acciones y exige un nivel de recursos que demanda un esfuerzo presupuestal adicional importante;

6. si todas las personas afectadas por el mismo problema acudieran a la acción de tutela para obtener la protección de sus derechos, se produciría una mayor congestión judicial.

Ciertamente, estos casos hito de la Corte Constitucional han repercutido en la actuación del resto de poderes, toda vez que se han llevado a cabo acciones de tipo legislativo como la Ley 1448 de 2011, así como acciones de naturaleza administrativa, tales como la expedición del Decreto 250 de 2005, por medio del cual se creó el "Plan Nacional para la Atención Integral a la Población Desplazada por la Violencia”; el Decreto 1660 de 2007 que reglamentó parcialmente el artículo 19 de la Ley 387 de 1997; el Decreto 790 de 2012, entre otros.

Con todo lo anteriormente expuesto, me encuentro en posibilidad de aseverar que la función desempeñada por la Corte Constitucional de Colombia corresponde 
al de una autoridad que busca, en la medida de sus posibilidades, cumplir con las obligaciones que derivan de los derechos humanos. Si bien es cierto que aún existe un largo camino por recorrer en la consolidación del Estado social y democrático de derecho; también es cierto que las acciones de este poder como regulador y verificador de una serie de medidas que exigen la actuación mancomunada del resto de los poderes estatales, da visos de una posible transformación de la realidad por la que hoy se transita.

\section{Hacia una incorporación de indicadores estructurales, de proceso y de resultado para la garantía de los derechos humanos}

Como podemos observar, hoy más que nunca en un Estado que adopta como modelo a la democracia constitucional, cobra vigencia la necesidad de una participación coordinada de los poderes estatales para alcanzar los fines sociales, entre ellos la garantía del amplio catálogo de derechos humanos que se han reconocido, pero no materializado. Siendo entonces, que aún y cuando la división de poderes se muestra cada vez más tenue, no por ello, nos alejamos de los principios que persigue la democracia; es decir, si la sociedad evoluciona, el poder, así como su división y control tienden también a evolucionar.

En la Conferencia Mundial de Derechos Humanos, celebrada en Ginebra, Suiza, del 19 al 30 de abril de 1993, se llevó a cabo un seminario internacional que tuvo como foco de atención lo relativo a los derechos económicos sociales y culturales -dimensión sustancial de la democracia constitucional-, en el que se hizo hincapié en la necesidad de incorporar indicadores para medir la progresividad de esta naturaleza de derechos. De tal manera que se precisó lo siguiente:

181. Para promover la realización progresiva de los derechos económicos, sociales y culturales es necesario tratar de lograr los siguientes objetivos:

a) aclarar el contenido de derechos concretos;

b) aclarar la naturaleza de las obligaciones de los Estados Partes;

c) que los Estados desarrollen planes para promover la realización progresiva de cada derecho;

d) mejorar la evaluación y vigilancia de la realización progresiva;

e) identificar y tratar las violaciones;

f) llegar a un mayor grado de cooperación dentro del sistema de las Naciones Unidas;

g) facilitar la participación de las organizaciones no gubernamentales y de las comunidades interesadas en cada una de las tareas anteriormente esbozadas;

h) aplicar metodologías científicas basadas en las estadísticas (Comité de Derechos Económicos, Sociales y Culturales, 1993). 
Como se puede advertir, la implementación de indicadores de los derechos prestacionales resulta ser fundamental, toda vez que sirve para aclarar el contenido de tales derechos; delimitar las obligaciones de los Estados; la evaluación permanente del acceso a estos, y en términos generales, el diseño e implementación de estrategias que permitan que la sociedad pueda gozar de tales derechos de manera efectiva; es decir, no solo nos dan un punto de partida, sino que además nos permiten trazar una ruta y evaluarla de manera constante con el objetivo de materializar los contenidos constitucionales y convencionales de los que ya se ha escrito en líneas que anteceden.

Sirve de ejemplo, la precisión que realiza la Oficina del Alto Comisionado de las Naciones Unidas:

La demanda y el uso de indicadores en el ámbito de los derechos humanos forman parte de un proceso más amplio y sistemático de aplicación, vigilancia y realización de los derechos. Junto con los planes nacionales de acción en materia de derechos humanos, estudios de referencia y enfoques basados en derechos respecto del desarrollo y la buena gobernanza, la labor de supervisión de los mecanismos de derechos humanos de las Naciones Unidas y de las instituciones regionales y nacionales de derechos humanos, los indicadores son un instrumento concreto y práctico para promover la realización de los derechos humanos y medir su aplicación (2012).

Por su parte, la Comisión Interamericana de Derechos Humanos (2008) estableció unos lineamientos para la elaboración de indicadores de progreso en materia de derechos económicos, sociales y culturales, en los que especifica la naturaleza y alcance de los siguientes indicadores:

1. Estructurales: buscan calcular qué medidas dispone el Estado para implementar los derechos del Protocolo de San Salvador, cómo se organiza el aparato institucional y el sistema legal del Estado y las medidas adoptadas para la garantía de los derechos.

2. De proceso: buscan medir la calidad y magnitud de los esfuerzos del Estado para implementar los derechos, evaluando las estrategias, planes, programas; vigilando directamente la aplicación de las políticas públicas.

3. De resultado: buscan medir el impacto real de las estrategias, programas, intervenciones del Estado. Proveen una medida cuantitativamente comprobable y comparable de la actuación del Estado en materia de realización progresiva de los derechos.

Como podemos observar, estos tres indicadores: estructurales, de proceso y de resultado, suelen ser imprescindibles, tanto para la delimitación del contenido de un derecho, así como para la precisión de las obligaciones por parte del Estado, incluso si estas corresponden a un solo órgano o institución estatal, o bien, a una diversidad de estos. Permiten diseñar de manera objetiva las acciones que tiendan a la garantía efectiva de los derechos, optimizando los recursos, sobre todo en aquellos derechos que implican una prestación por parte del Estado; es decir, a partir de los indicadores podemos administrar 
de manera correcta los recursos económicos destinados a la satisfacción de derechos, tales como la salud, la educación, vivienda e inclusive, alimentación.

Ahora bien, bajo el modelo de Estado de democracia constitucional en el que cobra especial relevancia la garantía de los derechos prestacionales, así como los rasgos en común que guardan los países latinoamericanos en su dimensión normativa, permiten afirmar - como ha quedado demostrado- que se está construyendo un derecho común en materia de derechos humanos, lo que nos permite llevar a cabo diálogos continuos entre jurisdicciones y demás poderes, incluyendo a la propia sociedad-sobre todo la organizada-. En ese mismo sentido, la figura del estado de cosas inconstitucional desarrollada por la Corte Constitucional de Colombia puede adoptarse en la región por los las altas Cortes, para resolver los problemas de tipo estructural, así como la incorporación de indicadores estructurales, de proceso y de resultado, como herramientas de evaluación de la efectividad de las resoluciones judiciales que vinculan al resto de los poderes, bajo un modelo de control de constitucionalidad y convencionalidad justificado en el propio desarrollo democrático.

\section{CONCLUSIONES}

Somos testigos de la evolución conceptual del Estado moderno, el cual ha ido ajustándose de acuerdo con su complejo contexto contemporáneo; sin que tal circunstancia haya provocado el abandono de la esencia liberal de finales del siglo XVIII. A mi parecer, resulta un perfeccionamiento, una puntualización a nivel teórico de las dimensiones que el Estado mismo implica a día de hoy. La democracia constitucional representa un modelo integral del Estado, ya que al considerar sus dimensiones formales y sustanciales desarrolla obligaciones ineludibles por parte de las autoridades, tendientes a alcanzar el verdadero orden social por el cual fue creado el Estado y dotado de poder, para servir a la comunidad y no para servirse de esta.

Atendiendo a la adopción del modelo de Estado denominado democracia constitucional, he de afirmar que hoy más que nunca, cobra vigencia la necesidad de una participación coordinada de los poderes estatales para alcanzar los fines sociales, entre ellos la garantía del amplio catálogo de derechos humanos que han reconocido, pero no materializado. Es decir, aún y cuando la división de poderes se muestra cada vez más tenue, no por ello, nos alejamos de los principios que persigue la democracia; si la sociedad evoluciona, el poder, así como su división y control tienden también a evolucionar. Si se observa con detenimiento, el poder y los derechos representan una simbiosis, por lo que no deben de entenderse a estos elementos con cierto grado de antagonismo. Ciertamente existe un sistema de pesos y contrapesos vigente, pero distinto al concebido hace dos siglos, toda vez que existen espacios de diálogo y de construcción de lo que he denominado "tétrada democrática" conformada por los poderes ejecutivo, legislativo, judicial y la sociedad. Sin embargo, esta tendencia de diálogo está dando sus primeros pasos en América Latina, toda vez que cada uno 
de los poderes establecidos presenta cierto rechazo a una aparente invasión de sus competencias, aún y cuando tales intervenciones tiendan a fortalecer al propio Estado.

Para hacer frente a los problemas estructurales que tienen lugar en nuestro país, tales como la crisis del sistema penitenciario, el acceso a los servicios de salud, el reciente desplazamiento forzado interno, la discriminación y desigualdad, la desaparición forzada, entre otros, una sentencia estructural representaría una respuesta adecuada a estos, siempre y cuando se tome en cuenta no solo la necesidad de fundamentación y motivación, sino además la implementación de indicadores estructurales, de progreso y, finalmente, de resultado, toda vez que servirán para aclarar el contenido de tales derechos violados; la correcta evaluación permanente del acceso a estos, la adecuada delimitación de las obligaciones estatales; y en términos generales, el diseño e implementación de estrategias que permitan que la sociedad pueda gozar de tales derechos de manera efectiva. Es decir, no solo nos dan un punto de partida, sino que además nos permiten trazar una ruta y evaluarla de manera constante con el objetivo de materializar los contenidos constitucionales y convencionales de los que ya se ha escrito en líneas que anteceden. De ahí que mi propuesta se desarrolle en el sentido de un concepto de Justicia dialógica multidireccional, cuyo centro del proceso dialógico sea el máximo tribunal nacional.

Hoy más que nunca, debemos asumir una postura pro derechos humanos ante un país en crisis, en una región que se caracteriza por ser la más desigual en el mundo. Los instrumentos internacionales ya se encuentran vigentes, la jurisprudencia interamericana sigue desarrollándose día a día; solo nos falta formar a verdaderos defensores del orden desde la sede más importante de transformación social, la academia; para con ello, hacer frente a las decisiones injustas tomadas por el poder.

\section{REFERENCIAS}

Bobbio, N. (1989). Estado, gobierno y sociedad. Por una teoría general de la política. México: Fondo de Cultura Económica.

Bobbio, N. (2003). El futuro de la democracia. México: Fondo de Cultura Económica. Bobbio, N. (2005). Teoría general de la política. Madrid: Trotta.

Comisión Interamericana de Derechos Humanos. (2008). Lineamientos para la elaboración de indicadores de progreso en materia de derechos económicos, sociales y culturales. Organización de los Estados Americanos, Washington, D.C.

Comité de Derechos Económicos, Sociales y Culturales. (1993). Informe del Seminario sobre los indicadores apropiados para medir los avances en la realización progresiva de los derechos económicos, sociales y culturales, A/ CONF.157/PC/73. Organización de las Naciones Unidas, Ginebra.

Ferrajoli, L. (2004). Derechos y garantias. La ley del más débil (4a ed.). Madrid: Trotta. 
Ferrajoli, L. (2011). Poderes salvajes. La crisis de la democracia constitucional. Madrid: Trotta.

Ferrajoli, L. (2013). Democracia y garantismo (2a ed.). Trotta.

Ferrajoli, L. (2014). La democracia a través de los derechos. Madrid: Trotta.

Ferrajoli, L., Bovero, M. (2012). Teoría de la democracia. Dos perspectivas comparadas. México: Instituto Federal Electoral.

Fiss, O. (2007). El derecho como razón pública. Madrid: Marcial Pons.

Fukuyama, F. (2004). La construcción del Estado. Hacia un nuevo orden mundial en el siglo XXI. Argentina: Ediciones B.

Gargarella, R. (2014). El nuevo constitucionalismo dialógico frente al sistema de los frenos y contrapesos. En R. Gargarella. Por una justicia dialógica. El poder judicial como promotor de la deliberación democrática (pp. 121-122). Argentina: Siglo XXI.

Garzón, E. (2003). El papel del poder judicial en la transición a la democracia. Isonomía: Revista de Teoría y Filosofía del Derecho (18), 32.

González, M. (2013). La tensión entre las Cortes constitucionales y otros poderes del Estado. En J. C. Henao (Ed.). Diálogos constitucionales de Colombia con el mundo. VII Encuentro de la Jurisdicción Constitucional (pp. 348-349). Bogotá: Universidad Externado de Colombia.

Guastini, R. (2001). Estudios de teoría constitucional. México: Fontamara.

Heller, H. (2015). Teoría del Estado. México: Fondo de Cultura Económica.

Huertas, O. (2019). Principio de legalidad penal y justicia transicional en colombia: legalidad estricta o legalidad flexible. Bogotá: Grupo Editorial Ibáñez.

Huertas, O., \& Morales, I. R. (2013). El sistema de responsabilidad penal para adolescentes: la expansión de la punibilidad en el neopunitivismo colombiano. Revista Guillermo de Ockham, 11(2).

Huertas, O., Amaya, C., \& Malte, G. D. (2013). Autoría mediata a través de aparatos organizados de poder. Tras la sombra del mando fugitivo: del dominio y la instrumentalización del delito. Opinión Jurídica, 81-98.

Huertas, O., Torres, H., \& Díaz, N. C. (2011). El leviatán de los mass media, el peligro de la otredad y el derecho penal: La construcción mediática del enemigo. Revista de Derecho, 96-117.

Huneeus, A., Couso, J., \& Rachel, S. (2010). Cultures of Legality: Judicialization and Political Activism in Contemporary Latin America. En A. Huneeus, J. Couso, \& S. Rachel (Eds.). Cultures of Legality judicialization and political activism in Latin America (pp. 3-4). New York: Cambridge University Press.

Jellinek, G. (2003). La Declaración de los Derechos del Hombre y del Ciudadano. México: Instituto de Investigaciones Jurídicas - UNAM.

Kelsen, H. (1992). Compendio de Teoría General del Estado. México: Colofón.

Lasalle, F. (2009). ¿Qué es una constitución? México: Grupo Editorial Tomo. 
Lutz, D. S. (2006). Principles of Constitutional Design. New York: Cambridge University Press.

Montesquieu. (2015). Del espíritu de las leyes. México: Porrúa.

Oficina del Alto Comisionado de las Naciones Unidas para los Derechos Humanos. (2012). Indicadores de derechos humanos. Guía para la medición y la aplicación. Nueva York: ONU.

Pegoraro, L. (2011). Derecho constitucional comparado. Itinerarios de investigación. Bogotá, D.C.: Universidad Libre.

Rodríguez, C. (2014). El activismo dialógico y el impacto de los fallos sobre derechos sociales. En R. Gargarella (Ed.). Por una justicia dialógica: El Poder Judicial como promotor de la deliberación democrática (pp. 212-213). Siglo XXI.

Rolla, G. (2002). Derechos fundamentales, Estado democrático y justicia constitucional. México: Instituto de Investigaciones Jurídicas - UNAM.

Rousseau, D. (2013). Las relaciones conflictuales entre las cortes constitucionales y los poderes ejecutivo, legislativo y judicial, expresión de una figura nueva de la democracia. En J. C. Henao (Ed.). Diálogos constitucionales de Colombia con el mundo. VII Encuentro de la Jurisdicción Constitucional (p. 324). Bogotá: Universidad del Externado de Colombia.

Sentencia T-025/04 (Corte Constitucional de Colombia 17 de junio de 2004).

Sentencia T-153-98 (Corte Constitucional de Colombia 28 de abril de 1998).

Serna, J. M. (2012). Impacto e implicaciones constitucionales de la globalización en el sistema jurídico mexicano. México: Instituto de Investigaciones Jurídicas, UNAM.

Von Bogdandy, A. (2013). Ius constitutionale commune latinoamericanum una aclaración conceptual desde una perspectiva europea. En L. Pérez, \& D. Valades. El Constitucionalismo Contemporáneo (pp. 49-53). México: Instituto de Investigaciones Jurídicas UNAM.

Zagrebelsky, G. (2005). El derecho dúctil (6 $6^{\mathrm{a}}$ ed.). Madrid: Trotta. 